\title{
Changes in Tinnitus by Cochlear Implantation: A Parametric Study of the Effect of Single-Electrode Stimulation
}

\author{
Francka J.J. Kloostra ${ }^{a, b}$ Emile de Kleine ${ }^{a, b}$ Rolien H. Free ${ }^{a, b}$ Rutger Hofman ${ }^{a, b}$ \\ Pim Van Dijk ${ }^{a, b}$
}

aDepartment of Otorhinolaryngology and Head and Neck Surgery, University Medical Center Groningen, Groningen, The Netherlands; ${ }^{\mathrm{b}} \mathrm{Graduate}$ School of Medical Sciences, Behavioural and Cognitive Neurosciences, University of Groningen, Groningen, The Netherlands

\section{Keywords}

Tinnitus · Cochlear implantation · Electrical stimulation

\begin{abstract}
Introduction: While cochlear implantation may have a positive effect on tinnitus, it is not effective in reducing tinnitus in all patients. This may be due to different patients requiring different strategies of electrical stimulation in order to obtain a positive effect on tinnitus. It is, therefore, important to identify the most effective stimulation strategies to reduce tinnitus. The simplest possible strategy is stimulation by only one electrode. In this study, we investigated tinnitus suppression by electrical stimulation via a single electrode of the cochlear implant. Methods: We performed a listening experiment in 19 adult participants, who had received a unilateral cochlear implant $(\mathrm{Cl})$ because of severe bilateral hearing loss. All of these patients had indicated that they suffered from tinnitus. During a 300-s interval, patients listened to blocks of single-electrode stimulation and rated the loudness of the stimulus and any effects on their tinnitus. The 300 -s interval included a block of single-electrode stimulation (duration $120 \mathrm{~s}$ ). In consecutive intervals, the stimulus differed in its cochlear location (basal or apical), its pulse rate
\end{abstract}

karger@karger.com www.karger.com/aud

Karger $\frac{1}{\%}$

GOPEN ACCESS
(C) 2020 The Author(s)

Published by S. Karger AG, Basel

This article is licensed under the Creative Commons AttributionNonCommercial-NoDerivatives 4.0 International License (CC BYNC-ND) (http://www.karger.com/Services/OpenAccessLicense). Usage and distribution for commercial purposes as well as any distribution of modified material requires written permission.
( 720 or $725 \mathrm{~Hz}, 1,200 \mathrm{~Hz}$, and 2,400 or 2,320 Hz), and amplitude (just above threshold or equivalent to moderate loudness). Thus, $2 \times 3 \times 2=12$ stimulus conditions were tested in each participant, and each condition was presented only once. During the experiment, the participants promptly rated the loudness of the stimuli and the loudness of their tinnitus on a Visual Analogue Scale (10-point VAS). Results: Significantly more tinnitus reduction was observed with stimuli at a moderate intensity level (30\%) compared to stimuli at near-threshold level $(18 \%)\left(x^{2}[1, N=222]=14.115, p<0.01\right)$. No significant differences in tinnitus levels resulted from the different pulse rates and stimulation sites. Eight participants reported an increase of tinnitus loudness under at least one stimulus condition. Changes in tinnitus loudness were generally minor, and never exceeded 3 points on the VAS. The overall effect of cochlear implantation on tinnitus, that is, the effect with full-array stimulation, was not correlated with the effectiveness of the single-electrode stimulation on tinnitus. Conclusion: In conclusion, the effect of single-electrode stimulation on tinnitus is relatively insignificant in comparison to the effect of full-array stimulation. However, in some individual cases, sustained single-electrode stimulation may be beneficial for tinnitus management.

(C) 2020 The Author(s)

Published by S. Karger AG, Basel

Francka J.J. Kloostra

Department of Otorhinolaryngology and Head and Neck Surgery

University Medical Center Groningen

Postbus 30.001, bb21 Hanzeplein 1, NL-9700 RB Groningen (The Netherlands)

f.j.j.kloostra@umcg.nl 


\section{Introduction}

A wide variety of approaches has been explored to suppress tinnitus by electrical stimulation. Stimulation has been applied via electrodes placed at various anatomical locations in the central or in the peripheral auditory system. Stimulation sites included the mastoid bone, the tympanic membrane, the promontory, the round window, and via a cochlear implant in the scala tympani [Graham and Hazellf, 1977; Portmann et al., 1979; Aran and Cazals, 1981; Dobie et al., 1986; Shulman, 1987; Jastreboff and Hazell, 1993; Okusa et al., 1993; Watanabe et al., 1995; Konopka et al., 2001; Rubinstein et al., 2003; Zeng et al., 2011; Kleine Punte et al., 2013; Arts et al., 2015a, b, 2016; Mertens et al., 2018]. The stimulation strategies also vary substantially, using direct current (DC) or alternating current (AC). Evidence suggests that the closer the active electrode is situated to the neural and sensory elements of the cochlea, the better the results are [Portmann et al., 1979; Aran and Cazals, 1981; Dobie et al., 1986]. Direct current stimulation causes more often more complete tinnitus suppression than alternating current stimulation [Portmann et al., 1979; Aran and Cazals, 1981].

Among the various approaches using electrical stimulation to suppress tinnitus, cochlear implantation is the most successful so far [Quaranta et al., 2008; Van De Heyning et al., 2008; Akdogan et al., 2009; Pan et al., 2009; Bovo et al., 2011; Zeng et al., 2011; Kleine Punte et al., 2013; Arts et al., 2015a, b, 2016; Mertens et al., 2018] Kleine Punte et al. [2013] demonstrated that full-length stimulation is the most effective manner to reduce tinnitus in cochlear implant patients. However, not all such patients benefit from full-length stimulation and it remains necessary to search for other stimulation strategies to suppress tinnitus using a CI [Tyler, 1994; Mo et al., 2002; Di Nardo et al., 2007; Akdogan et al., 2009; Pan et al., 2009; Bovo et al., 2011; Arts et al., 2012; Kompis et al., 2012; Arts et al., 2015a, b]. In the study of Kleine Punte et al. [2013], only stimuli at the basal electrodes were tested regarding a reduction of tinnitus by (single) electrode stimulation. Other previous studies also only partially explored single-electrode stimulation. In this study, we broadened the parameter space by using a large number of possible stimulation configurations via a cochlear implant, both in terms of the cochlear electrode location, stimulation rate, and amplitude. We aimed to systematically explore the various parameters of single-electrode stimulation and investigate their effect in a relatively large group of participants. If these single-electrode stimuli are effective in tinnitus reduction, they could potentially be added to full-electrode stimulation for speech comprehension in patients who do not experience tinnitus reduction by full-electrode stimulation alone.

We describe a parametric study of tinnitus suppression, using electrical stimulation by means of a CI, where just one electrode of the cochlear implant array was activated. The purposes of this study were to investigate (1) whether single-electrode stimulation can reduce tinnitus loudness and (2) whether tinnitus suppression with single-electrode stimulation depends on the electrode location, stimulation pulse rate, or stimulation amplitude.

\section{Materials and Methods}

\section{Subjects}

For this study, all CI patients who were implanted at the University Medical Center Groningen in the period from 2000 until 2009 received a letter asking for their participation. All were 18 years of age or older and were implanted at least 6 months before the current set of experiments. Among those who positively reacted to our letter, there were $19 \mathrm{CI}$ patients who as adults received a unilateral cochlear implant because of bilateral severe hearing loss (standard of care in the Netherlands) and had suffered from subjective, chronic, and constant tinnitus in daily life for more than 3 months. The current study was not aimed at reducing the tinnitus handicap. Rather, we were interested in reducing the tinnitus percept irrespective of the handicap the tinnitus possibly posed. Hence, both subjects with low and high handicap scores (THI or THQ) and with all kinds of effects of full-length stimulation through the CI on their tinnitus (tinnitus onset, no effect on tinnitus, and tinnitus reduction) qualified for participation. Patients were implanted with CI from the companies Cochlear Inc. (implant types CI24R CA, CI24R CS, and CI24RE CA) or Advanced Bionics Inc. (Hi Res 90K Helix). The study was approved by the Medical Ethical Committee of the University Medical Center Groningen and informed consent obtained from all subjects.

\section{Study Design}

A listening experiment was performed, during which patients were presented with an electrical pulse train via a single CI electrode. The stimuli were generated by clinical software and presented via the clinical sound processor of the patient while connected to the standard clinical interface.

The software allowed for the presentation of blocks of $5 \mathrm{~s}$ of single-electrode stimulation; 24 blocks combined to 120 s. Subjects indicated the loudness of the stimulus and the loudness of their tinnitus on a Visual Analogue Scale (0-10, with 0 being inaudible and 10 being very loud). Subjects were instructed to indicate the loudness every $15 \mathrm{~s}$ and to rate their tinnitus regardless of its laterality. Loudness rating started $60 \mathrm{~s}$ prior to the stimulus presentation. Next, a single electrode was activated for $120 \mathrm{~s}$, and finally 
Fig. 1. The raw traces of the VAS for both the stimulus and tinnitus of patient number 5 using a stimulus at the apical electrode, with a pulse rate of $1,200 \mathrm{~Hz}$ and the stimulus at C-level. The arrows mark the duration of the stimulus.
Stimulus

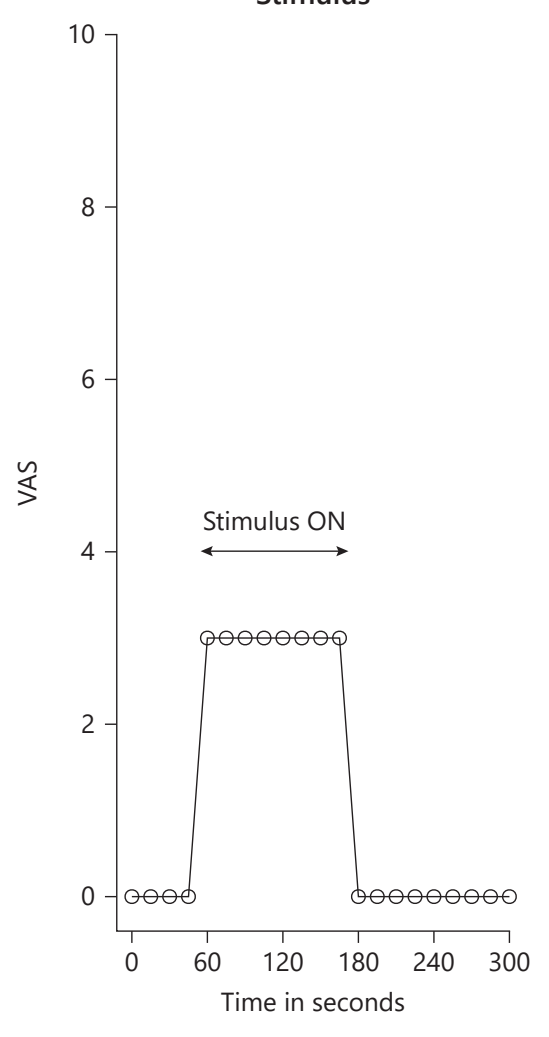

Tinnitus

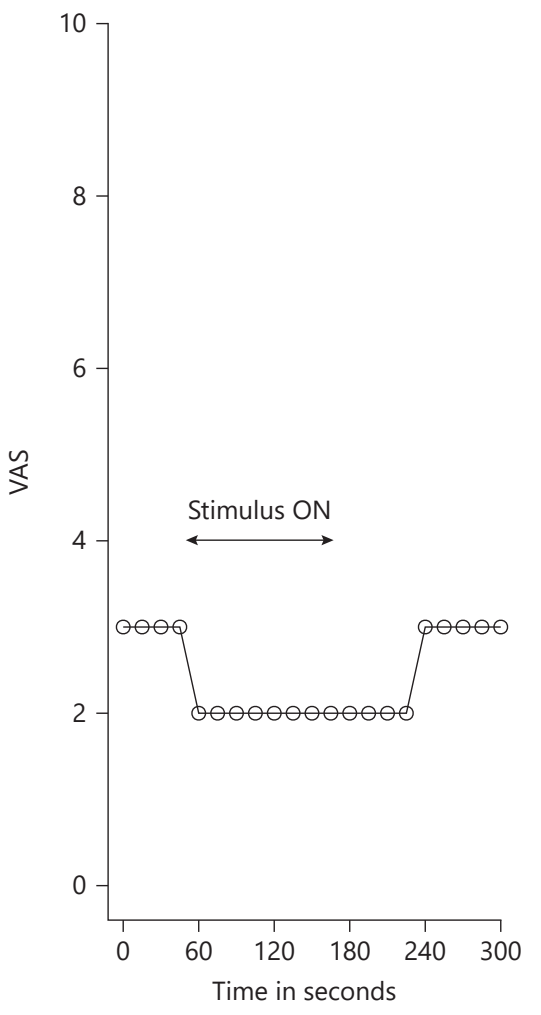

there were $120 \mathrm{~s}$ without stimulus. Thus, loudness estimates were acquired for a total of $60+120+120=300 \mathrm{~s}$.

A total of 12 stimulus conditions were tested, during which (1) the position of stimulation in the cochlea, (2) the stimulation rate, and (3) the stimulation amplitude were varied. The electrode position was either a basal or an apical electrode. For devices of the Cochlear Co., the electrode array consisted of 22 electrodes. The activated basal and apical electrodes, respectively, were chosen to be \#3 and \#20. For Advanced Bionics devices, the electrode array contained 16 electrode contacts, which are labelled from apex to base. The basal and apical electrodes used for stimulation were \#14 and \#3, respectively. The pulse rates were 720 (low), 1,200 (medium), and 2,400 (high) Hz for Cochlear and 725 (low), 1,200 (medium) and 2,320 (high) $\mathrm{Hz}$ for Advanced Bionics. The amplitude of the stimulation pulses was set to correspond to a level that was either just audible, or was clearly perceived and judged to be of moderate loudness by the participant. These levels were termed $\mathrm{T}$ (for threshold) or C (for comfortable), respectively, and established for each patient just prior to the experiment. In the subsequent text, we refer to the near-threshold level as the T level and about the moderate intensity level as the $\mathrm{C}$ level.

With 2 different locations of stimulation, 3 different pulse rates, and 2 different amplitude levels, the total number of stimulus conditions was 12 . Thus, with $300 \mathrm{~s}$ per stimulus, the full experiment lasted $60 \mathrm{~min}$, excluding frequent breaks. The order in which the stimuli were presented to the patients was randomized. Two pa- tients did not complete the entire experiment, due to tiredness and concentration problems. Of these patients, one received 10 stimuli (patient 1 ) and the second 8 stimuli (patient 2).

\section{Descriptive Variables}

Besides using the VAS as the primary outcome measure, we collected data on the lateralization of tinnitus, the anamnestic effect of the cochlear implant on tinnitus in daily life (full-array stimulation), and the postoperative tinnitus handicap scores by means of questionnaires (Tinnitus Handicap Inventory [THI]; Tinnitus Handicap Questionnaire [THQ]) which were completed just prior to the experiment.

\section{Statistical Analysis}

To describe the effect on tinnitus loudness, we counted the number of stimulus conditions for which the tinnitus either decreased, remained unchanged, or increased. Tinnitus was scored "decreased" when the mean score on the VAS of the tinnitus during stimulation was reduced by at least 1 point compared to the mean score on the VAS prior to the stimulus. An "increase" of tinnitus was scored when the mean score on the VAS during stimulation increased at least 1 point compared to the mean score on the VAS prior to stimulation. No effect was scored when the mean score on the VAS remained stable, or was reduced/increased less than 1 point during stimulation. $\chi^{2}$ tests were used to assess whether there was a significant influence of electrode position, pulse rate, 
Table 1. Patient characteristics and in the rightmost 3 columns the effect of single-electrode stimulation

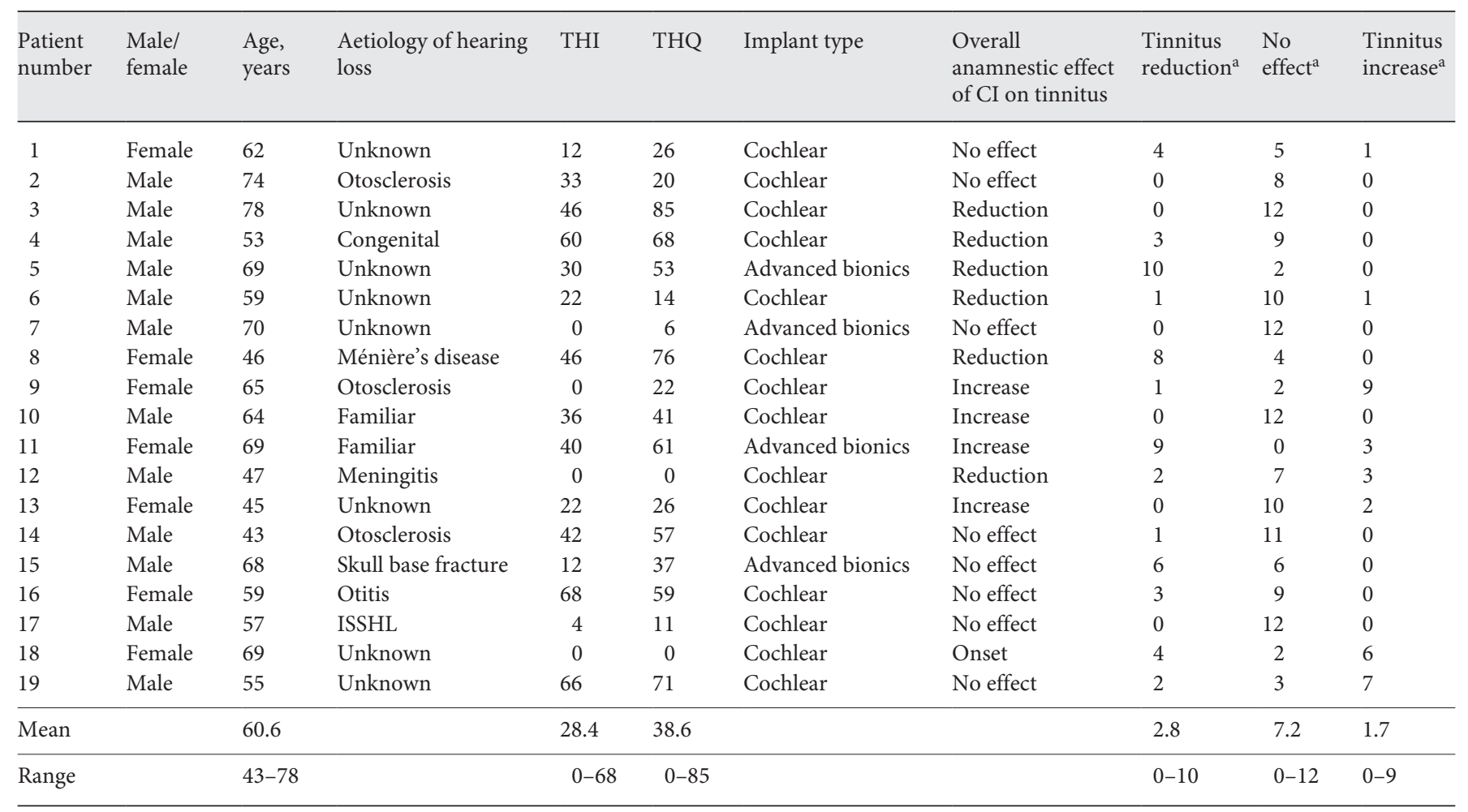

Patients 1 and 2 did not complete the entire experiment. THI, Tinnitus Handicap Inventory; THQ, Tinnitus Handicap Questionnaire; CI, cochlear implant. ${ }^{a}$ Number of conditions with the indicated effect of single-electrode stimulation.

or level of stimulation on the decrease of tinnitus during stimulation. Statistical significance was defined as $p<0.05$, using SPSS 20.0 to perform the analyses. A non-parametric Mann-Whitney U test was used to assess whether there was a significant difference between the tinnitus handicap scores of the patients who experienced tinnitus suppression and the patients who did not.

\section{Results}

We included 7 females and 12 males; the age of the patients ranged from 43 to 78 years at the time of the experiments. Figure 1 shows an example of the raw traces of the VAS for both the stimulus and tinnitus of a patient with a stimulus from the apical electrode, with a medium pulse rate and a moderate intensity level. The stimulus rating was 0 on the VAS at the beginning of the experiment, increased to 3 during the stimulus, and returned to 0 after the stimulus offset. Simultaneously, the VAS of the tinnitus started at 3 , decreased to 2 during the stimulus, and remained at 2, until $45 \mathrm{~s}$ after the stimulus offset. Eventually, the tinnitus returned to its original loudness.
The stimulus VAS trace in Figure 1 is representative for the stimulus VAS traces of all patients obtained during this experiment. The stimuli at low intensity level (T-level) (mean VAS 1.1, SD 0.2) were rated lower compared to the stimuli at moderate intensity level (C-level) (mean VAS 5.0, SD 0.5). The traces of the VAS of the tinnitus varied greatly across patients and stimulus conditions. In 9 patients, tinnitus returned to its pre-stimulus level immediately after the stimulus ended. In 8 patients, in $50 \%$ of the conditions that caused a reduction of the tinnitus, the tinnitus remained suppressed up to $240 \mathrm{~s}$ (end of trial).

Table 1 shows patient characteristics, causes of hearing loss, the scores of the tinnitus handicap questionnaires, implant type, the overall anamnestic effect of cochlear implantation on tinnitus, and the effect of stimuli for each subject. The spread of both the postoperative THI and THQ scores was large. Thirteen out of 19 patients $(68 \%$, Table 1$)$ experienced a reduction of their tinnitus in one or more stimulus conditions. Eight participants out of 19 (42\%) reported an increase of tinnitus loudness in at least one stimulus condition. There were 


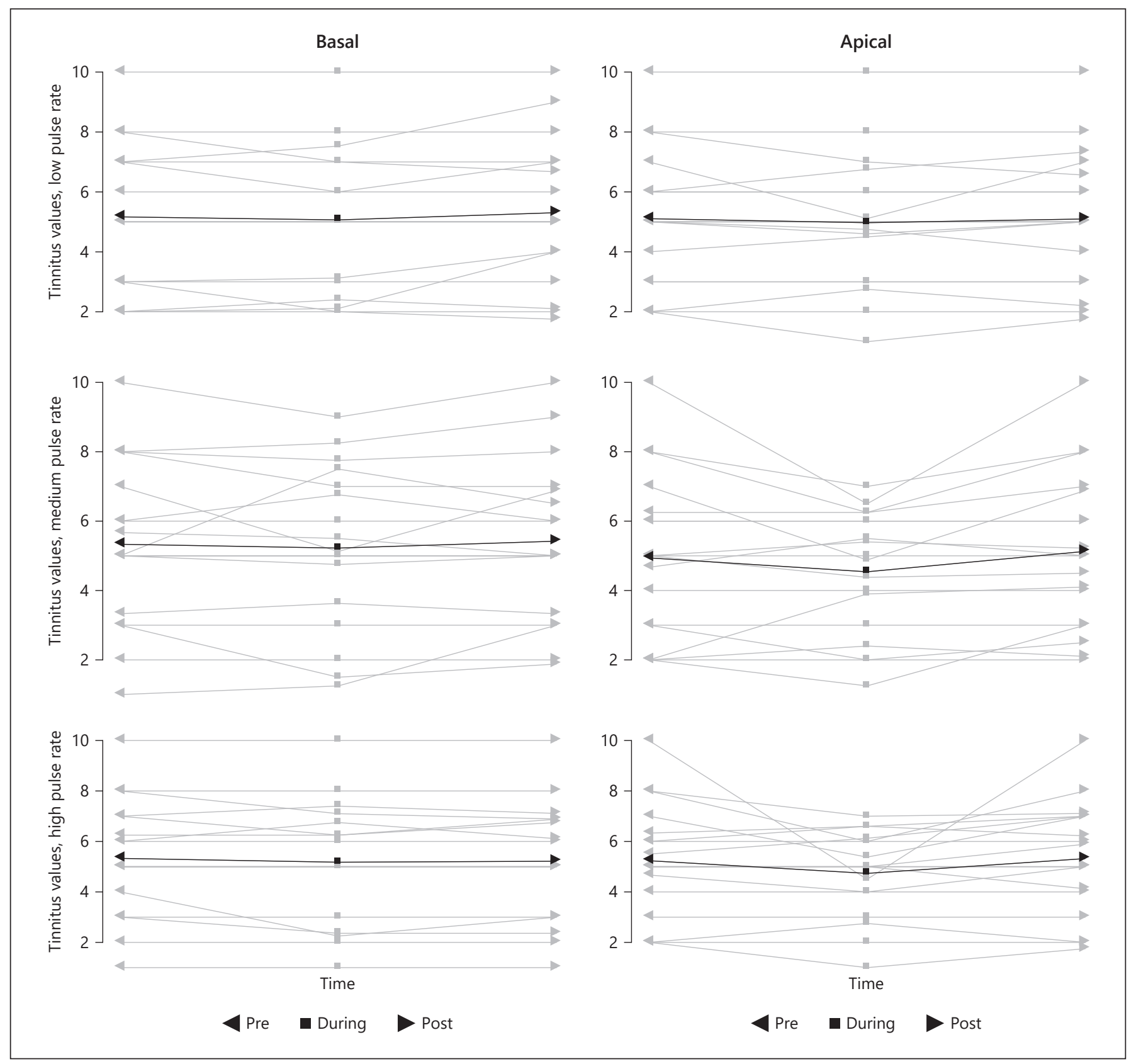

Fig. 2. Patient response traces together with a mean trace for all stimuli at $C$ level. Each thin line represents the result for an individual subject. The thick line shows the mean across subjects. The $y$ axis represents the mean tinnitus VAS and the $x$ axis represents the phases during the experiment: pre-stimulus, during stimulus, and poststimulus.

no significant differences in the postoperative THI and THQ scores between patients with a decrease of tinnitus (THI 36.2, SD 23.1; THQ 49.5, SD 21.3) with single-electrode stimulation and the patients without a decrease of tinnitus (THI 23.5, SD 18.4; THQ 31.5, SD 28.9) with single-electrode stimulation. Three out of the 4 patients with an Advanced Bionics implant and 10 of the 15 patients who used a Cochlear implant experience a decrease of tinnitus with single-electrode stimulation. Five out of the 8 patients with no anamnestic effect on their tinnitus of full-array stimulation, as determined by the questionnaire, experienced tinnitus reduction of single-electrode 
stimulation. Three of the 5 patients with an overall increase of tinnitus by full-array stimulation experienced tinnitus reduction from single-electrode stimulation. Thus, some patients who experienced no effect or even a negative effect of anamnestic full-array stimulation on tinnitus experienced a positive effect of single-electrode stimulation on tinnitus.

Figure 2 shows all individual patient traces together with a mean trace for all conditions at the C-level. Only the C-level results are shown, since they gave the most obvious results, that is, those obtained by stimulation at moderate intensity. To produce the traces, we calculated 3 mean VAS values; one for the measurements before stimulation, one for the measurements during the stimulation, and one for the measurements after stimulation. The mean effect for all stimuli is shown to be minimal. Stimulation of the apical electrode with medium and high pulse rates showed the most prominent effects.

For the various stimulus conditions, Figure 3 shows the number of patients for whom tinnitus decreased, remained unchanged, or increased. Altogether, a reduction of the tinnitus amplitude of at least 1 point on the VAS was observed in $24 \%$ of the stimulus presentations. The size of the changes was generally small, and never exceeded 3 points on the VAS. A tinnitus reduction was more commonly observed when stimulation was at the moderate intensity level (30\% of stimulus conditions) compared to stimulations at the low intensity level $(18 \%)\left(\chi^{2}[1, N=\right.$ $222]=14.115, p<0.01)$. Tinnitus reduction was also more commonly observed when stimulation was at medium (26\%) or high $(29 \%)$ pulse rates compared to stimulation with low (16\%) pulse rates, but this effect was not significant $\left(\chi^{2}[2, N=222]=1.413, p=0.493\right)$. Concerning the location of the stimulation, stimulation at apical electrodes (29\%) more often resulted in a positive effect compared to stimulation at basal electrodes (19\%), which, again, was not a significant difference $\left(\chi^{2}[1, N=222]=\right.$ $0.136, p=0.712$ ).

\section{Discussion/Conclusion}

This study examined the acute effect of single-electrode stimulation via a CI on tinnitus. For most stimulus conditions, patients did not experience any change in their tinnitus and the effects of single-electrode stimulation on tinnitus varied substantially across patients. A systematic evaluation of the dependence of tinnitus suppression on the rate, amplitude, or cochlear location of the stimulus showed a significant effect of amplitude

Changes in Tinnitus by Single-Electrode

Stimulation only, in that stronger electrical stimulation tended to result in greater tinnitus suppression.

In our study, a reduction of at least 1 point on the VAS was observed in $24 \%$ of the stimulus presentations. Within these $24 \%$, the size of the changes was in general small and never exceeded 3 points on the VAS. The VAS is a clinical measuring scale that has been established as valid and reliable in a range of clinical and research applications [Wewers and Lowe, 1990]. Thus, the question arises as to how reliable and reproducible these changes are. In this respect, most patients who indicated that there was a tinnitus reduction also indicated this reduction to be consistent during the whole stimulus and even after the stimulus. To filter out small, inconsistent, and irrelevant changes, we calculated a mean VAS score for the period before, during, and after the stimulus.

Several studies examined the effects of electrical stimulation through a CI on tinnitus using stimuli that did not encode environmental sounds. There are four major studies that performed experiments similar to ours.

The first study by Rubinstein et al. [2003] presented high-rate pulse train stimuli at various stimulus intensities to 3 CI patients. In one of these 3 patients, stimulation had no effect on the tinnitus. In 1 patient, tinnitus was only suppressed during the stimulus, and a further patient showed suppression of tinnitus during as well as after the stimulus.

The second study by Chang and Zeng [2012] used multiple electrical stimuli in $13 \mathrm{CI}$ patients. These stimuli differed in pulse rate, place of stimulation, and level of stimulation. Their most important finding was that the effect of electrical stimulation from a cochlear implant is very subject specific and that regardless of the kind of stimulus some subjects were more sensitive for experiencing tinnitus reduction. In addition, they found that louder stimuli more often produced a positive effect compared to softer or sub-threshold stimuli. There was also a small effect showing that loud sounds produced by a higher pulse rate give more positive results compared to loud sounds from low pulse rates.

In a third study, Arts et al. [2015b] presented electrical stimuli in the cochleae of 10 CI patients. The stimuli differed in amplitude, location, modulation, polarity, and stimulation rate. They also found that the effect of CI electrical stimulation on tinnitus was subject specific and that stimulations that caused a medium-to-loud sound more often resulted in a positive effect. Furthermore, they found no relationship between the effect of full-length electrical stimulation, that is, encoding speech, on tinnitus on the one hand and the effect of electrical stimulation 


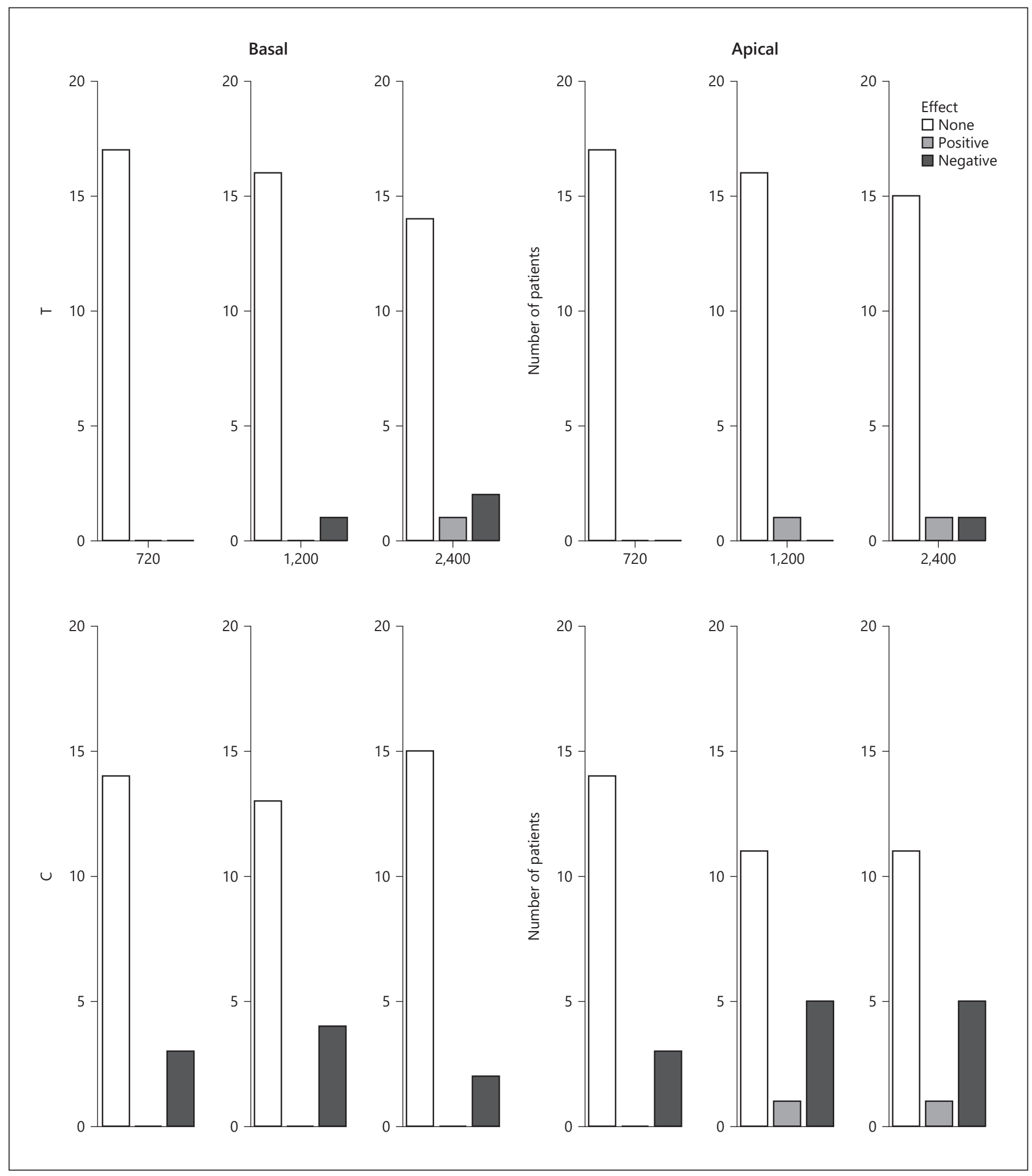

Fig. 3. The number of patients who perceived an increase or decrease of the tinnitus loudness for each of the stimulus conditions. 
with single-electrode stimulation or stimulation with looped patterns, that is, without encoding speech, on the other.

The final study by Kleine Punte et al. [2013] used 7 subjects with severe unilateral sensorineural hearing loss and implanted with a CI, because of tinnitus. In the first weeks after implantation, they only activated the most basal electrodes and found no significant tinnitus reduction. Tinnitus reduction only occurred when all electrodes were activated. In line with these results, we also found smaller tinnitus reduction from stimulation at the basal electrodes compared to stimulation at the apical electrodes.

Altogether, the results from all studies are compatible with our results; the effect of single-electrode stimulation through a CI on tinnitus is subject specific and is not related to the effect of full-length stimulation through a cochlear implant, and stimulation with higher amplitudes more often results in a positive effect on tinnitus. The fact that stimuli with a larger amplitude more often cause a positive effect on tinnitus suggests that the tinnitus reduction is generally based on masking, similar to the way in which acoustic tinnitus maskers can mask tinnitus. The sound introduced by the cochlear implant can reduce the contrast between the tinnitus signal and the background activity in the auditory system, with a decrease of the patients' perception of their tinnitus [Vernon, 1977]. Obviously, a stimulus with a relatively large amplitude will not result in complete silence. The maximum effect of such a stimulus is to replace the tinnitus percept by the perception of the masking sound. Thus, the aim of a masking therapy can be to establish an overall sound percept that a patient finds more acceptable than the tinnitus alone [Vernon and Meikle, 2003].

In our study, we investigated the acute effects of singleelectrode stimulation on tinnitus. The effects were rather small and not correlated with the effect of the CI on tin- nitus in daily life. In other words, there is a difference between the acute effects as observed in the current experiments and the chronic effects on tinnitus by daily use of the CI. Arts et al. [2016] found that a tinnitus-reducing stimulation pattern added to a speech-coding stimulation strategy can be implemented without adverse effects on speech discrimination. To establish whether single-electrode stimulation may also have a similar clinical benefit on tinnitus, chronic studies with long-term single-electrode stimulation would be required.

\section{Conclusions}

Single-electrode stimulation through a CI can ameliorate tinnitus in some implant users. Chronic studies would be required to establish the benefit of such an approach for tinnitus suppression. Such studies would presumably explore single-electrode stimulation for tinnitus suppression combined with full-electrode stimulation for speech comprehension.

\section{Statement of Ethics}

The study was approved by the Medical Ethics Committee of the UMCG. All included patients have given their written informed consent.

\section{Conflict of Interest Statement}

There are no financial interests to disclose. There are no conflicts of interest.

\section{Funding Sources}

There are no funding sources.

\section{References}

Akdogan O, Ozcan I, Ozbek C, Dere H. Tinnitus after cochlear implantation. Auris Nasus Larynx. 2009;36(2):210-2.

Aran JM, Cazals Y. Electrical suppression of tinnitus. Ciba Found Symp. 1981;85:217-31.

Arts RAGJ, George ELJ, Stokroos RJ, Vermeire K. Review: cochlear implants as a treatment of tinnitus in single-sided deafness. Curr Opin Otolaryngol Head Neck Surg. 2012;20(5): 398-403.
Arts RAGJ, George ELJ, Chenault MN, Stokroos RJ. Optimizing intracochlear electrical stimulation to suppress tinnitus. Ear Hear. 2015a; 36(1):125-35.

Arts RA, George EL, Griessner A, Zierhofer C, Stokroos RJ. Tinnitus suppression by intracochlear electrical stimulation in single-sided deafness: a prospective clinical trial: part 1 . Audiol Neurotol. 2015b;20(5):294-313.
Arts RA, Georde EL, Griessner A, Zierhofer C, Stokroos RJ. Long-term effects of intracochlear electrical stimulation with looped patterns on tinnitus: a case study. Ear Nose Throat J. 2016;95(4-5):E9-15.

Bovo R, Ciorba A, Martini A. Tinnitus and cochlear implants. Auris Nasus Larynx. 2011; 38(1):14-20.

Chang JE, Zeng F-G. Tinnitus suppression by electric stimulation of the auditory nerve. Front Syst Neurosci. 2012;6:19. 
Di Nardo W, Cantore I, Cianfrone F, Melillo P, Scorpecci A, Paludetti G. Tinnitus modifications after cochlear implantation. Eur Arch Otorhinolaryngology. 2007;264(10):1145-9.

Dobie RA, Hoberg KE, Rees TS. Electrical tinnitus suppression: a double-blind crossover study. Otolaryngol Neck Surg. 1986;95(3 Pt 1):31923.

Graham JM, Hazell JW. Electrical stimulation of the human cochlea using a transtympanic electrode. Br J Audiol. 1977;11(2):59-62.

Jastreboff PJ, Hazell JW. A neurophysiological approach to tinnitus: clinical implications. $\mathrm{Br}$ J Audiol. 1993;27(1):7-17.

Kleine Punte A, De Ridder D, Van De Heyning P. On the necessity of full length electrical cochlear stimulation to suppress severe tinnitus in single-sided deafness. Hear Res. 2013;295: 24-9.

Kompis M, Pelizzone M, Dillier N, Allum J, Demin N, Senn P. Tinnitus before and 6 months after cochlear implantation. Audiol Neurotol. 2012;17(3):161-8.

Konopka W, Zalewski P, Olszewski J, OlszewskaZiaber A, Pietkiewicz P. Tinnitus suppression by electrical promontory stimulation (EPS) in patients with sensorineural hearing loss. Auris Nasus Larynx. 2001;28(1):35-40.
Mertens G, Van Rompaey V, Van de Heyning P. Electric-acoustic stimulation suppresses tinnitus in a subject with high-frequency singlesided deafness. Cochlear Implants Int. 2018; 19(5):292-6.

Mo B, Harris S, Lindbœek M. Tinnitus in cochlear implant patients: a comparison with other hearing-impaired patients. Int J Audiol. 2002; 41(8):527-34.

Okusa M, Shiraishi T, Kubo T, Matsunaga T. Tinnitus suppression by electrical promontory stimulation in sensorineural deaf patients. Acta Otolaryngol. 1993;501:54-8.

Pan T, Tyler RS, Ji H, Coelho C, Gehringer AK, Gogel SA. Changes in the tinnitus handicap questionnaire after cochlear implantation. Am J Audiol. 2009;18(2):144-51.

Portmann M, Cazals Y, Negrevergne M, Aran JM. Temporary tinnitus suppression in man through electrical stimulation of the cochlea. Acta Otolaryngol. 1979;87(3-4):294-9.

Quaranta N, Fernandez-Vega S, D'Elia C, Filipo $\mathrm{R}$, Quaranta A. The effect of unilateral multichannel cochlear implant on bilaterally perceived tinnitus. Acta Otolaryngol. 2008; 128(2):478-85.

Rubinstein JT, Tyler RS, Johnson A, Brown CJ. Electrical suppression of tinnitus with highrate pulse trains. Otol Neurotol. 2003;24(3): 478-85.
Shulman A. External electrical tinnitus suppression: a review. Am J Otol. 1987;8(6):479-84.

Tyler RS. Advantages and disadvantages expected and reported by cochlear implant patients. Am J Otol. 1994;15(4):523-31.

Van De Heyning P, Vermeire K, Diebl M, Nopp P, Anderson I, De Ridder D. Incapacitating unilateral tinnitus in single-sided deafness treated by cochlear implantation. Ann Otol Rhinol Laryngol. 2008;117(9):645-52.

Vernon J. Attempts to relieve tinnitus. J Am Audiol Soc. 1977;2(4):124-31.

Vernon JA, Meikle MB. Masking devices and alprazolam treatment for tinnitus. Otolaryngol Clin North Am. 2003;36(2):307-20.

Watanabe K, Okawara D. [Electrocochleographic analysis of the suppression of tinnitus by electrical promontory stimulation and changes in compound action potentials]. Nihon Jibiinkoka Gakkai Kaiho. 1995;98(8):1310-7.

Wewers ME, Lowe NK. A critical review of visual analogue scales in the measurement of clinical phenomena. Res Nurs Health. 1990;13:22736.

Zeng FG, Tang Q, Dimitrijevic A, Starr A, Larky J, Blevins NH. Tinnitus suppression by lowrate electric stimulation and its electrophysiological mechanisms. Hear Res. 2011;277:616. 